\title{
Protocol Defined Response Criteria
}

National Cancer Institute

\section{Source}

National Cancer Institute. Protocol Defined Response Criteria. NCI Thesaurus. Code C126042.

A description of the disease response to treatment as characterized by the clinical trial protocol. 